\title{
The Current Status in Treatment of Depressive or Anxiety Disorders During Pregnancy with Antidepressants
}

\author{
Faruk Uguz* \\ Associate Professor, Department of Psychiatry, Meram Faculty of Medicine, Konya University, Konya, Turkey
}

Pregnancy is one of those important medical conditions during which more specific evaluations and increased attention towards the treatment of patients by physicians are needed. Although the prevalence of psychiatric disorders during pregnancy appears to be neutral, in reality, (kaynak:Arch gen), approximately one fifths of pregnant women experience a depressive or anxiety disorder [1,2]. Psychopharmacology, which is one of main components in the treatment of depressive and anxiety disorders during this period, is therefore an important issue.

Studies reported in the literature suggest that antidepressant usage has shown a significant increase during the pregnancy period $[3,4]$. However, the available studies have some methodological limitations. For example, studies based on automated databases and retrospective chart review mostly lack information such as timing, duration and dosage of antidepressant exposure. In addition, research analyses generally do not include the potential impact of non-iatrogenic confounders on the fetus and pregnancy [5]. Moreover, studies with large sample size comparing women untreated and treated with antidepressants who have depressive or anxiety disorder with similar severity are very inadequate. For these reasons, it is unclear whether treatment of maternal depressive or anxiety disorder with antidepressanst is safe and more beneficial with respect to the development of the fetus compared with untreated maternal illness.

Taking into consideration the results of the available studies, treatment of mild or moderate depressive disorders with antidepressants appears not to be superior from untreated maternal illness for fetus development and pregnancy termination. On the other hand, the effects of some psychotherapy methods, particularly cognitive-behavioral therapy, on depressive and anxiety disorders have been well documented. Therefore, psychiatrists should carefully evaluate the level of usefulness of any pharmacological treatment of pregnant women, and these decisions should be made in consultation with the patient and her relatives. Although no studies in the published literature have examined these factors, psychopharmacological treatment of depressive or anxiety disorders during pregnancy period may be appropriate in the following conditions: 1) The presence of a serious depressive and anxiety disorder or their comorbidity leading to severe loss in occupational area and social or family relationships of mothers. 2) The presence of severe weight loss and metabolic disturbances in mothers secondary to the psychiatric disorder. 3) The existence of a high suicidal risk in mother. 4) The existence of additional medical conditions (e.g., hypertension, gestational diabetes mellitus) which uncontrolled due to the psychiatric disorder. 5) The existence of a temporal relationship between the onset of intrauterine growth restriction of the fetus and the onset of maternal psychiatric disorders.

\section{References}

1. Vesga-López O, Blanco C, Keyes K, Olfson M, Grant BF, et al. (2008) Psychiatric disorders in pregnant and postpartum women in United States. Arch Gen Psychiatry 65: 805-815.

2. Uguz F, Gezginc K, Kayhan F, Sari S, Buyukoz D (2010) Is pregnancy associated with mood and anxiety disorders? A cross-sectional study. Gen Hosp Psychiatry 32: 213-215.

3. Andrade SE, Raebel MA, Brown J, Lane K, Livingston J, et al. (2008) Use of antidepressant medications during pregnancy: a multisite study. Am J Obstet Gynecol 198: 194.e1-194.e5.

4. Alwan S, Reefhuis J, Rasmussen SA, Friedman JM; National Birth Defects Prevention Study (2011) Patterns of antidepressant medication use among pregnant women in a United States population. J Clin Pharmacol 51: 264-270.

5. Gentile S (2011) Selective serotonin reuptake inhibitor exposure during early pregnancy and the risk of birth defects. Acta Psychiatr Scand 123: 266-275.
*Corresponding author: Faruk Uguz, Associate Professor, Department of Psychiatry, Meram Faculty of Medicine, Konya University, Akyokuş, 42080, Konya, Turkey, Tel. +90 332223 6306; E-mail: farukuguz@gmail.com

Received March 26, 2012; Accepted March 28, 2012; Published April 03, 2012

Citation: Uguz F (2012) The Current Status in Treatment of Depressive or Anxiety Disorders During Pregnancy with Antidepressants. J Clinic Case Reports 2:e114. doi:10.4172/2165-7920.1000e114

Copyright: (C) 2012 Uguz F. This is an open-access article distributed under the terms of the Creative Commons Attribution License, which permits unrestricted use, distribution, and reproduction in any medium, provided the original author and source are credited. 